\title{
Enhanced Solubility and Dissolution Rate of Aceclofenac by Using Spray Drying Techniques
}

\author{
Sahilhusen I Jethara* and Mukesh R Patel \\ ${ }^{1}$ Department of Pharmaceutics, Shri B. M. Shah College of Pharmaceutical Education \& Research, Gujarat, India
}

\begin{abstract}
The present study deals with improvement in aqueous solubility of aceclofenac using HPMC K-15M, PVP-K30 and Eudragit RS-100 in varying ratio by spray-drying at optimized condition. The samples of solid dispersion were subjected to fourier transform infra-red spectroscopy (FTIR), differential scanning calorimetry (DSC), powder X-ray diffraction morphology (PXRD), scanning electron microscopy (SEM), in vitro drug release and stability studies. $A$ decrease in the melting enthalpy in DSC indicates conversion of crystalline to amorphous state. The results were supported by PXRD, FTIR and SEM data revealed a characteristic decrease in crystallinity. The dissolution studies demonstrated a marked increase in the dissolution rate in comparison with pure drug. The considerable improvement in the dissolution rate of aceclofenac from spray drying, decreased drug crystallinity, altered surface morphology and micronization. The optimized co-crystals exhibited excellent stability on six month storage at accelerated conditions of $40^{\circ} \mathrm{C}$ and $75 \%$ relative humidity. Thus, the solid dispersion obtained by spray drying is a potential tool for improving drug solubility in water.
\end{abstract}

Keywords: Aceclofenac; Spray drying; Solid dispersion; Polymers; Solubility

\section{Introduction}

Today, $35-40 \%$ all new chemical entities suffer from poor aqueous solubility. The poor solubility and low dissolution rate of poorly water soluble drugs in the aqueous gastro-intestinal fluids often cause insufficient bioavailability [1]. And therefore Improvement of the solubility of poorly water-soluble drugs is one of the most challenging aspects in formulation development. The model drug mentioned is practically water insoluble. It belongs to BCS class II and its bioavailability dissolution dependent. Due to poor aqueous solubility of the model drug it is chosen as a drug candidate to improve its solubility and bioavailability [2]. Various techniques are followed to improve solubility of drug. Preparation of Solid Dispersion of drug is one of the techniques to enhance solubility.

\section{Objective}

The Objective of present study was to formulate spray dried solid dispersion of Aceclofenac to improve aqueous solubility and drug release.

\section{Experimental Methods}

\section{Preparation of solid dispersion}

The melting point of drug is $154^{\circ} \mathrm{C}$ and polymer i.e. PVP $\mathrm{K}-30$, HPMC K-15M and Eudragit RS-100 are in the range of $145-155^{\circ} \mathrm{C}$ [2]. Hence hot melt technique was used for the preparation of solid dispersion of Aceclofenac drug. The drug Aceclofenac in combination with different polymer include PVP-K30, Eudragit RS100 and HPMC $\mathrm{K}-15 \mathrm{M}$ in different ratio as mentioned in Table 1 was heated on a laboratory scale heating mantle in a porceline dish at a temperature of $70^{\circ} \mathrm{C}$ to obtain a physical complex of Aceclofenac and polymers [3]. The batches obtained were coded as I, II, and III respectively.

\section{Spray drying method}

Aceclofenac in combination with PVP-K30, HPMC K-15M and Eudragit RS-100 in different ratio were dissolved in sufficient amount of different solvent to obtain clear solutions [4]. Spray drying was carried out using laboratory scale spray dryer at optimized condition.

\begin{tabular}{|l|c|c|c|}
\hline \multirow{2}{*}{\multicolumn{1}{|c|}{ Polymer }} & \multicolumn{3}{|c|}{ Drug-Polymer Ratio } \\
\hline PVP-K30 & I & II & III \\
\hline Eudragit RS-100 & $1: 1$ & $1: 1.5$ & $1: 2$ \\
\hline HPMC K-15M & $1: 1$ & $1: 1.5$ & $1: 2$ \\
\hline
\end{tabular}

\section{Result and Discussion}

Solid dispersions were successfully evaluated such as drug content, saturation solubility, scanning electron microscopy (SEM), differential scanning calorimeter (DSC), X-ray powder diffraction, FTIR spectral analysis for plain drug indicate sharp peak for halide group at 740-750 $\mathrm{cm}^{1}$. Peak at $1253 \mathrm{~cm}^{-1}$ are for aryl $-\mathrm{NH}_{2}$ group attached to aromatic ring, $2942 \mathrm{~cm}^{-1}$ indicate $\mathrm{COOH}$ - group, while region 1452-1500 $\mathrm{cm}^{-1}$ indicates $\mathrm{C}=\mathrm{C}$ stretching for aromatic carbons. These all peaks were also seen in the SDs indicating no change in structural characteristic of the drug molecule or no interaction with polymer after spray drying. SDs of Drug: Eudragit RS-100 ratio of 1:1 showed higher dissolution. SDs of Drug: HPMC K-15M showed higher dissolution in the ratio of 1:1. SDs of Drug: PVP-K30 showed higher dissolution in the ratio of 1:1.5. Accelerated stability study was found that there was minute decrease in drug content after 6 month. Drug release profile and solubility remained unchanged.

\section{Conclusion}

The first phase of study involved preparation and evaluation of

*Corresponding author: Sahilhusen I. Jethara, Department of Pharmaceutics, Shri B. M. Shah College of Pharmaceutical Education \& Research, Modasa-383315, Gujarat, India, Tel: +91-2774-249587; E-mail: sahil.pharm4@gmail.com

Received December 17, 2014; Accepted January 04, 2015; Published June 06, 2015

Citation: Jethara SI, Patel MR (2015) Enhanced Solubility and Dissolution Rate of Aceclofenac by Using Spray Drying Techniques. Intel Prop Rights 3: 140. doi:10.4172/2375-4516.1000140

Copyright: (c) 2015 Jethara SI, et al. This is an open-access article distributed under the terms of the Creative Commons Attribution License, which permits unrestricted use, distribution, and reproduction in any medium, provided the original author and source are credited. 
Citation: Jethara SI, Patel MR (2015) Enhanced Solubility and Dissolution Rate of Aceclofenac by Using Spray Drying Techniques. Intel Prop Rights 3: 140 . doi: $10.4172 / 2375-4516.1000140$

Page 2 of 2

various solid dispersions of Aceclofenac using different polymers as Eudragit E-100, HPMC K-15M, and PVP-K30. Improving the solubility and dissolution characteristics of poorly water-soluble drugs is important to achieve better bioavailability.

Stability studies of the selected formulations were done for 3 months with a sampling time of 0 days, 7 days, 15 days, 30 days and 90 days. The samples were evaluated by drug content, saturation studies, dissolution studies, FTIR studies.

1) Amongst all the solid dispersions prepared, solid dispersion prepared by spray drying method using PXM 188 as carrier and TPGS as surfactant was found to be more efficient in improving the drug solubility and dissolution rate of fenofibrate.

2) Drastic differences between solubility as well as dissolution behavior of pure drug and formulations.

3) TPGS showed drastic enhancement in the solubility.

4) FTIR characterization indicated that there was no drug-carrier chemical interaction.

5) DSC analysis indicated formation of a single phase solid dispersion in which the drug was dissolved into the matrix of the carrier.
6) PXRD studies suggested that there has been a large change in the crystalline structure of fenofibrate in the solid dispersions. It also indicated possibility of conversion of a large portion of fenofibrate from crystalline to amorphous form in the solid dispersions.

7) SEM studies revealed that significant change had occurred in the surface morphology of fenofibrate in the solid dispersions.

8) Stability studies demonstrated no physical, chemical or crystalline changes in the samples.

9) In-vivo studies in rat indicated increase in the anti-hyperlipidemic activity for solid dispersion with PXM 188 and TPGS prepared by spray drying method than the pure drug.

\section{References}

1. Carstensen JT (1995) Drug stability: Principle and practice. 2nd Edition, Marcel Dekker, New York 538-550.

2. Chiou WL, Rigelman S (1971) Pharmaceutical application of solid dispersion system. J Pharm Sci 60: 1281-1302.

3. Habib MJ (2000) Pharmaceutical solid dispersion Technology. Published by CRC Press, USA 1-25.

4. Paradkar A, Ambike AA, Jadhav BK (2004) Characterization of curcumin PVP solid dispersion obtained by spray drying. Int J Pharm 271: 281-286. 